\title{
A forward diversity index
}

\author{
Stephen Carley • Alan L. Porter
}

Received: 21 March 2011 / Published online: 30 September 2011

(C) Akadémiai Kiadó, Budapest, Hungary 2011

\begin{abstract}
We introduce an indicator to measure the diffusion of scientific research. Consistent with Stirling's 3-factor diversity model, the diffusion score captures not only variety and balance, but also disparity among citing article cohorts. We apply it to benchmark article samples from six 1995 Web of Science subject categories (SCs) to trace trends in knowledge diffusion over time since publication. Findings indicate that, for most SCs, diffusion scores steadily increase with time. Mathematics is an outlier. We employ a typology of citation trends among benchmark SCs and correlate this with diffusion scores. We also find that self-cites do not, in most cases, significantly influence diffusion scores.
\end{abstract}

Keywords Knowledge diffusion - Integration score - Diffusion score - Citation patterns · Self-citations - Subject category behavior

\section{Introduction}

Interdisciplinary research ("IDR") is widely hailed as desirable, even essential, to accomplish meaningful science. Chubin and Connolly (1982) showed that research constrained within disciplinary bounds can be inbred to the point of irrelevance. Many hold that generating important scientific advances and solving complex societal problems demands interdisciplinarity. We do not attempt a broad review of studies of interdisciplinary processes here, but note an active literature (c.f., Wagner et al. 2011; Klein 1996).

Wagner et al. (2011) pose key challenges to those who would measure interdisciplinary research, noting needs to address multiple facets, including:

\section{S. Carley $(\bowtie)$}

School of Public Policy, Georgia Institute of Technology, Atlanta, GA, USA

e-mail: stephen.carley@gmail.com

\section{A. L. Porter}

Technology Policy and Assessment Center, Georgia Institute of Technology, Atlanta, GA, USA

A. L. Porter

Search Technology Inc., Atlanta, GA, USA 
- Inputs, process attributes, and outputs

- Shorter and longer term impacts

- Social and cognitive phenomena

- Aggregate (global) and particular (local) features

Over the years, the community of scholars studying interdisciplinary research and how to facilitate it has identified many additional attributes of potential interest e.g., definitions, antecedent factors, interplay of organizational structures and IDR processes, funding and infrastructure roles, and network dynamics (Jurkovick and Paelincer 1984; Chubin et al. 1986; Porter et al. 2006; Stokols et al. 2003; Wagner et al. 2011). ${ }^{1}$ Notable current initiatives include strengthening knowledge about, and resources for, "team science." 2 This paper keys on the "impacts" of research as evidenced by citing patterns.

A key to this attention to IDR is the belief that it can make a difference-that IDR can contribute importantly to solving complex societal problems and generating meaningful innovations. That draws us toward the challenge prompting this indicator developmentcan we devise a useful measure of research knowledge diffusion? If so, that should aid in IDR assessment, as well as general study of research knowledge transfer processes. The "Diffusion score" that we present is an "impact" measure-i.e., it purports to address the influence of a piece, or body, of research. It is a bibliometric indicator based on citation patterns - with attendant strengths and weaknesses inherent in citation practices and data. Before turning to those matters, we describe the genesis of the Diffusion score, considering other diffusion approaches and the "Integration score."

A large amount of existing research on knowledge diffusion has focused on patent citation analyses (De Bellis 2009). According to De Bellis, such analysis provides "quantitative evidence of a complex character in matters of impact, quality, and knowledge diffusion." Citation analyses indicate that knowledge diffusion tends to occur first and foremost at the local level, and that knowledge spillovers can be and are hampered by international borders (De Bellis 2009). She also makes the point that there often exist a small number of patents that are cited time and again that take the "lion's share in the knowledge diffusion process." This handful of patents seems to command a disproportionate influence in driving knowledge diffusion.

Using network theory and visualization techniques, patent citation studies emphasize the growth and evolution of patent citation networks over time (Chen and Hicks 2004). Patent citation analyses have found that knowledge embedded in public research patents tends to diffuse more quickly than knowledge embedded in corporate patents (Bacchiocchi and Montobbio 2009). Similar studies also find that the knowledge diffusion process is asymmetric in that it tends to occur more rapidly within, than between, fields ( $\mathrm{Yu}$ et al. 2010). However, a 2010 study suggests that a number of research areas are converging, and the diffusion of knowledge across fields is a major force facilitating this process (Liu and Rousseau 2010). Li and Meng (2010) caution that patent and publication citation

\footnotetext{
1 "INTERSTUDY" was a professional association with such a focus that held a series of conferences and issued proceedings books (c.f., Birnbaum-More et al. 1990).

2 The "Science of Team Science" initiative is active with various resources, including an e-mail list (listserv@list.nih.gov), annual conference (//scienceofteamscience.northwestern.edu/scits-2011-conference), resources (e.g., Collaboration Team Science: A Field Guide at the National Institutes of Health - https://ccrod. cancer.gov/confluence/download/attachments/47284665/TeamScience_FieldGuide.pdf?version=2\&modific ationDate $=1285330231523$; a Team Science Toolkit website under development), ongoing research assessments - e.g., on transdisciplinary tobacco use research centers (TTURCs) (Masse et al. 2008; Stokols et al. 2003).
} 
processes, while sharing important features, also differ importantly-in particular, in the inherent purposes of citation.

Research on diffusion has found that physical and technological proximity enhances knowledge diffusion (MacGarvie 2005). For this reason, it has been argued that technology policy towards a given industry should take into account the role of inventor mobility in facilitating the flow of ideas across space and innovating organizations (Stolpe 2002). Similar studies point to the role of collaborative networks to measure knowledge flows within the context of geographic localization and firm boundaries (Singh 2005; AutantBernard et al. 2007). Results of these studies indicate that knowledge flows within regions or firms are stronger than those which cross regional or firm boundaries, but that boundary effects diminish once interpersonal relationships are accounted for (Singh 2005). Chen et al. (2009) presents an intriguing case that the pattern of citation to an article is predictive of special importance (e.g., future Nobel prizes). He makes the case that widespread, rapid citation in multiple fields is the indicator. Intrigued by this, we pursue our Diffusion measure for publications, based on citing patterns to them.

Different types of new knowledge can diffuse across different types of domains. Our Georgia Tech group has been pursuing a series of assessments of nanotechnology research patterns. In the "nano" context, we can distinguish the diffusion of various forms of knowledge, such as:

- A vision's influence-Drexler's (1986) tome that popularized nanotechnology as potentially world-changing, traceable through a bifurcated research community where some cited his work heavily and some avoided it (Porter and Cunningham 1995)

- Enabling technology - the scanning tunneling microscope that allowed researchers to see and manipulate molecular interactions (for which Binning and Rohrer received the 1986 Nobel Prize in Physics)

- New material forms - the buckyball, carbon nanotube, and graphene (2010 Nobel prize in Physics to Geim and Novoselov)

New knowledge can spread along many dimensions, such as:

- Across disciplines [our primary interest, with disciplines operationalized as Web of Science ("WOS") subject categories ("SCs")]

- Over time (of particular interest here as well)

- From science to technology (as evidenced, for instance, in patents citing non-patent literature)

- Through space (geographical diffusion-e.g., among countries, from "North to South")

- Among organizations (a special concern in intellectual property regimes)

Several studies have emphasized techniques with which to measure the "integration" of academic disciplines. These analyses seek to assess how an author or publication weaves previously disparate knowledge into one fabric. The "Integration score" calculates the diversity of a set of references based upon the journals in which they were published. It relies upon the WOS SCs, used to classify journals (Porter et al. 2007, 2008). For example, if a given publication cites multiple references from a broad array of academic disciplines it is said to be integrative. In that our diffusion scoring is an analogy to the Integration score, we expound upon that in the "Methodology" section.

Integration scores are backward-oriented from the perspective that they measure diversity among the publications upon which a given author or publication has drawn. The present inquiry moves in the opposite direction by introducing a metric for assessing diversity among publications that cite a given author or body of research. This metric 
incorporates Stirling's (2007) three concepts of diversity - variety (by accounting for the number of citing disciplines), balance (by accounting for the distribution of cites among disciplines), and disparity (by accounting for how similar or dissimilar these categories are). We measure knowledge diffusion based on publication citations. The diffusion score is forward-oriented in that it takes a given publication, author, or stream of research as its starting point and measures the propagation of knowledge from that vertex.

Our analyses illustrate the application of this metric to six benchmark WOS SCs to assess papers' degree of utility, influence, or popularity, among external disciplines. The six are:

- Biotechnology \& Applied Microbiology [Biotech]

- Engineering, Electrical \& Electronic [EE]

- Mathematics [Math]

- Medicine-Research \& Experimental [Med-R\&E]

- Neurosciences [Neurosci]

- Physics-Atomic, Molecular \& Chemical [Phy-AMC]

This benchmark dataset, which was first built and used by Porter and Rafols (2009a), was constructed in a manner consistent with the US National Academies' interests in science, engineering, and medicine. The analysts sought benchmark data on how interdisciplinary research is across a sample of fields, in support of evaluation of the National Academies Keck Futures Initiative [www.keckfutures.org]. They compiled samples at 10-year intervals (1975-2005). Sample size reflected tradeoffs to be large enough that Integration score averages would be robust, with manageable sampling effort. Sizes varied about an evolving target of $\sim 1,000$ publications per SC as some SCs contained fewer articles in particular years and some with more were easier to take all than to sample. Variety in terms of traditional versus emergent, and basic versus applied research, domains was a sampling criterion. Porter and Rafols (2009a) describe the sampling methodology used to build the above dataset in detail. Appendix A contains detailed descriptions of these 6 SCs from WOS.

As will become apparent in the research that follows, different SCs exhibit different levels of diffusion. The present study seeks to measure and explain these differences. Of particular interest is whether diffusion scores tend to increase over time since publication or level off asymptotically. Exploring differences in benchmark diffusion scores between fields yields insights into the diffusion process. Coming to terms with the forces underlying the process can advance understanding of research knowledge transfer processes.

\section{Methodology}

Since diffusion can be thought of as integration in reverse, a proper understanding of the measurement should be grounded in the basic mechanics of integration scoring. Drawing on a National Academies report (2005), integration can be defined as follows:

IDR is a mode of research by teams or individuals that integrates:

- perspectives/concepts/theories and/or

- tools/techniques and/or

- information/data

from two or more bodies of specialized knowledge or research practice. 
The WOS SCs suit this definition well (and are commensurate in scope with the examples offered by the National Academies study, such as "operations research").

The formula for integration is:

$$
I=1-\sum_{i, j} \mathrm{~s}_{i j} \mathrm{p}_{i} \mathrm{p}_{j}
$$

where $\mathrm{p}_{i}$ is the proportion of cited references corresponding to the SC $i$ in a given paper. The summation is taken over the cells of the paper's cited SC $x$ cited SC matrix. $s_{i j}$ is the cosine measure of similarity between SCs $i$ and $j$ for cross-citation in WOS for the year 2007. In their 2007 paper, Porter et al. considered using the Pearson correlation, but favored the cosine measurement instead, citing multiple studies on its goodness of fit with their newly introduced Integration measure. ${ }^{3}$ To maintain consistency with the Integration score, we likewise use the cosine identity:

$$
\frac{\sum_{i}\left(x_{i} y_{i}\right)}{\sqrt{\sum_{i} x_{i}^{2} \sum_{i} y_{i}^{2}}}
$$

where $x$ and $y$ represent observations (counts) used to calculate the cosine (i.e. these represent subject classifications within a correlation matrix that measures the distance between SCs).

To generate the $\mathrm{s}_{i j}$ values, Loet Leydesdorff obtains the WOS journal-to-journal crosscitation matrix from Thomson Reuters. He applies a thesaurus that associates each journal to one or more WOS SCs to convert this to an SC-to-SC citation matrix. Ismael Rafols factor analyzes that SC-to-SC cosine matrix from Leydesdorff to extract factors used in science overlay maps - 18 in the version that encompasses both science (Science Citation Index-SCI) and social science (Social Science Citation Index-SCI) (Rafols et al. 2010). They label those "Macro-Disciplines" (groups of SCs) to describe the group's emphasis, based on judgment. [See also discussion in the section on visualizing diffusion.]

We add a few cautionary notes. Our methods depend on the WOS Subject Categories. Publications in other than WOS-indexed journals are not addressed. Use of SCs as the unit of classification means that an article's journal, not its content, determines its categorization. Assignment of journals to SCs is based on a combination of citation patterns and editorial judgment at the Institute for Scientific Information (ISI)-it is not unambiguous. However, deviations tend to be "nearby"-i.e., the preferred SC is usually very close to the WOS-assigned one (in our science overlay maps), so analyses with large numbers (e.g. above 100 papers for a first approximation), such as presented in science overlay maps, are quite robust (Rafols and Leydesdorff 2009; Klavans and Boyack 2009). This does imply that fine distinctions should not be emphasized. Katz and Hicks discuss the difficulties inherent to achieving a standardized journal classification scheme and offer their own approach for classifying SCI journals (1995).

\footnotetext{
3 See notes 17 and 18 of Porter et al 2007-the version of the Integration score used in this reference was based on co-citation of SCs in a sample of some 30,000 WOS papers. Conceptually, co-citation is very appealing. However, this resulted in a relatively sparse (not so robust) similarity matrix. Since then we have changed to using cross-citation, as noted here. This has the advantages of a much larger sample (a full year of WOS publications) and heartier SC similarity matrix. As a result, however, the recalibrated Integration scores are considerably lower. The new scores correlate extremely highly with the earlier Integration scores (Porter and Rafols 2009a, b).
} 
Integration score corresponds to (1-Stirling's diversity measure) (Rafols and Meyer 2010). Integration scores range from 0 to 1 , with higher scores indicative of greater interdisciplinarity.

The diffusion score is analogous, with $\mathrm{p}_{\mathrm{i}}$ pertaining to the citing papers' SCs:

$$
D=1-\sum_{i, j} \mathrm{~s}_{i j} \mathrm{p}_{i} \mathrm{p}_{j}
$$

As is the case with Integration scores, Diffusion scores range from 0 to 1 , with higher scores indicative of greater infiltration into external disciplines. We illustrate by devising this metric for six benchmark SCs, derived from 1995 WOS publications (based on Porter et al. 2009). Table 1 (below) provides summary statistics for this dataset, including sample sizes and mean cited reference and times cited counts. A citation extraction macro, developed by the authors, was then applied to these benchmarks. After citing articles were downloaded, scores were calculated for each and used to inform much of the following discussion.

\section{Benchmarking integration versus diffusion scores}

Figure 1 provides a visual for mean Integration scores among the 1995 benchmark SCs. Integration scores were calculated for those publications with 3 or more CSC instances (i.e., this could be 3 cites of the same SC). We observe that Neuroscience and medical research and experimentation show the highest integration, followed closely by three SCs, while Math stands apart as the least integrative of the disciplines examined in this paper. These scores show modest increase over time, for benchmark samples in 1975, 1985, 1995, and 2005 (Rafols and Leydesdorff 2009).

Figure 2a provides mean aggregate diffusion scores for the 1995 benchmarks. This figure is a corollary to Fig. 1. We note, however, that unlike integration scores (which are based on a fixed list of cited references), diffusion scores can change over time. Hence, if we re-plotted Fig. 2a (which reflects citation data through the end of 2010) in ten years' time the influence of additional citations would most likely change its shape. Comparing this figure with its predecessor, we observe that diffusion scores are larger than integration scores for Biotech, EE, Med-R\&E and Neuroscience, while the converse is true for Math

Table 1 Mean Statistics for 1995 Benchmark SCs

\begin{tabular}{lrllllll}
\hline $\begin{array}{l}\text { Subject } \\
\text { category }\end{array}$ & $\begin{array}{l}\text { Sample } \\
\text { size }\end{array}$ & $\begin{array}{l}\text { Cited } \\
\text { refs. } \\
\text { (mean) }\end{array}$ & $\begin{array}{l}\text { Times } \\
\text { cited } \\
\text { (mean) }\end{array}$ & $\begin{array}{l}\text { Integration } \\
\text { score } \\
\text { (mean) }\end{array}$ & $\begin{array}{l}\text { Diffusion } \\
\text { score } \\
\text { (mean) }\end{array}$ & $\begin{array}{l}\text { Integration } \\
\text { versus cited } \\
\text { refs. (Pearson } \\
\text { correlation) }\end{array}$ & $\begin{array}{l}\text { Diffusion } \\
\text { versus times } \\
\text { cited (Pearson } \\
\text { correlation) }\end{array}$ \\
\hline Neuroscience & 1,910 & 42.53 & 43.46 & 0.43 & 0.46 & -0.05 & 0.04 \\
Med-R\&E & 664 & 33.65 & 59.72 & 0.42 & 0.47 & -0.07 & 0.10 \\
Physics-AMC & 1,017 & 33.40 & 32.52 & 0.40 & 0.38 & -0.10 & 0.09 \\
Biotech & 840 & 31.23 & 27.37 & 0.37 & 0.44 & -0.07 & 0.15 \\
EE & 1,719 & 18.40 & 13.51 & 0.35 & 0.37 & 0.24 & 0.14 \\
Math & 658 & 17.90 & 9.11 & 0.19 & 0.19 & 0.22 & 0.13 \\
Total & 6,808 & 30.43 & 30.54 & 0.37 & 0.40 & 0.20 & 0.13 \\
\hline
\end{tabular}




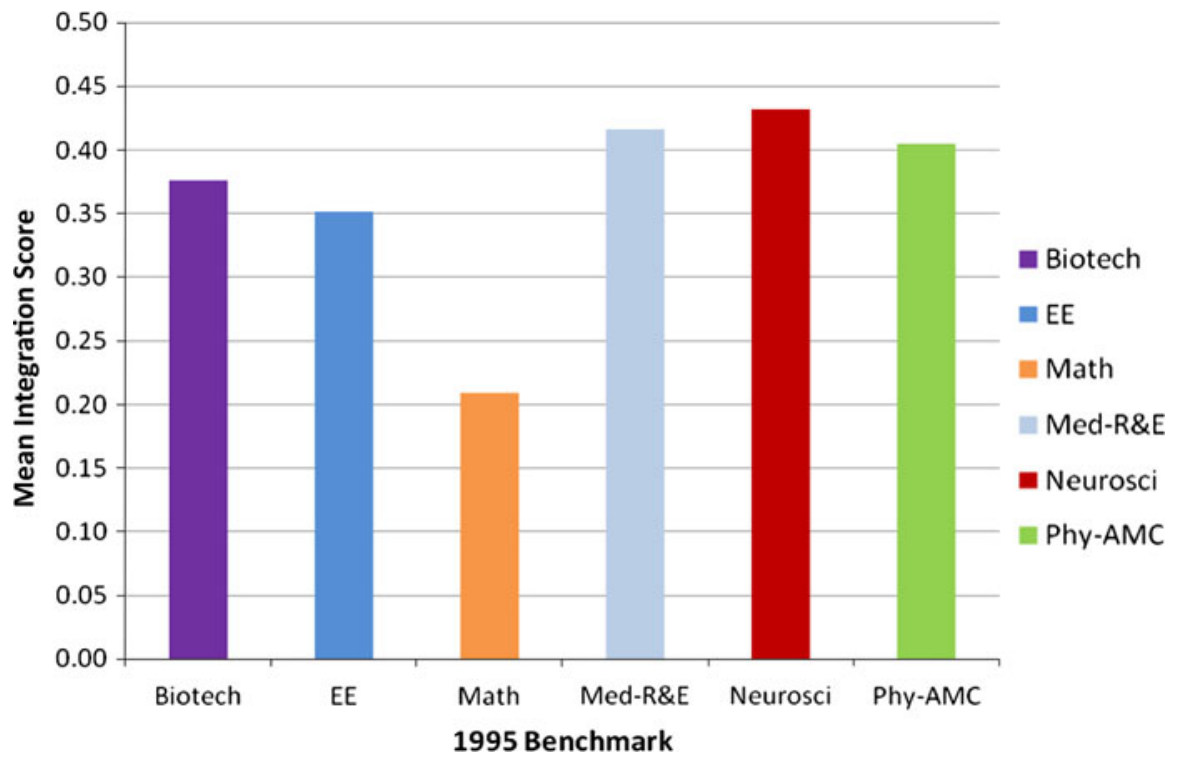

Fig. 1 Mean integration scores for 1995 benchmark sample articles

and Physics-AMC. As noted previously, however, diffusion scores are not static. Figure $2 \mathrm{~b}$, which plots diffusion scores by individual citing year, considers changes in these scores over time.

The scores in Fig. $2 \mathrm{~b}$ are calculated on an annual basis (i.e. citing articles were analyzed based on their publication year and results were therefore mutually exclusive, as opposed to cumulative). It will be emphasized that we only calculate Diffusion scores for those publications with 3 or more citing SC instances (CSCs). ${ }^{4}$ One reason for this rule is that our coverage of SCs is not perfect (i.e. while our journal-subject category thesaurus covers significant ground, and continues to improve, it does not capture every journal indexed by WOS). If a given publication was cited by a single SC, its diffusion score would be 0 . We make room for the possibility, however, that publications that our macro indicates are cited rarely may actually have more citations-they may have been referenced by external knowledge sources (e.g. dissertations, websites, conferences), or by articles in non-WOS journals or in a WOS journal not captured in our thesaurus. For this reason, we believe it to be the lesser of two evils to not calculate diffusion scores for articles with fewer than 3 CSCs instances (i.e., classifying a publication on the basis of 1 or 2 CSCs leaves an uncomfortable amount of room for deviation from its "true" Diffusion score). We note that the greater the number of CSCs instances, the smaller the expected deviation from a publication's true diffusion score becomes. However, if we set our CSC threshold too high, we drop an undesirably large number of publications from our analysis. We note also that this rule may result in a given publication being included in the mean annual diffusion

\footnotetext{
4 That is, as an extreme case, suppose that only one paper cites a given 1995 paper in the sample. If that citing paper appeared in a journal that WOS associates with 3 or more SCs, it would meet this criterion to be included. Or, suppose that a given 1995 paper were cited by exactly 3 papers in a given year, and that all 3 appeared in journals categorized in the same SC. This would also just reach the threshold of 3 instances (of one) SC.
} 

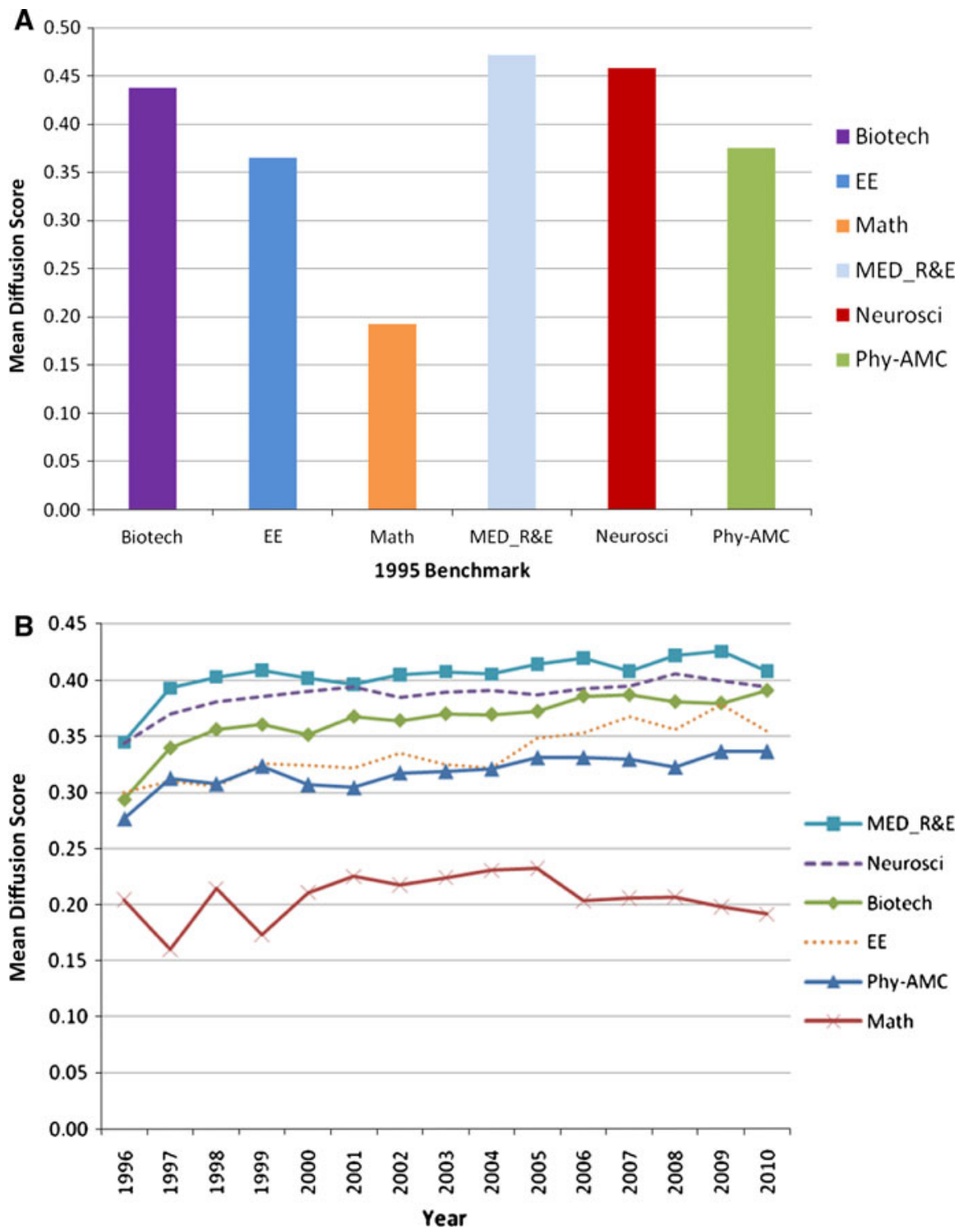

Fig. 2 a Annual diffusion scores for 1995 benchmark sample articles. b Mean annual diffusion scores for 1995 benchmark sample articles

score for year $x$, but not included in the mean annual diffusion score for a subsequent year. For an analysis of the percentage of publications with at least 3 CSCs instances, by benchmark, see Appendix B. We anticipate that most analyses would consolidate citing for all years, reducing sensitivity to the exclusion of papers cited fewer than 3 times.

We note from Fig. 2b that, as was the case in Fig. 1, Neuroscience and Medical R\&E assume leadership positions. Math is noticeably different from the other SCs analyzed. The difference in how knowledge flows for Math and other fields, a difference which can 


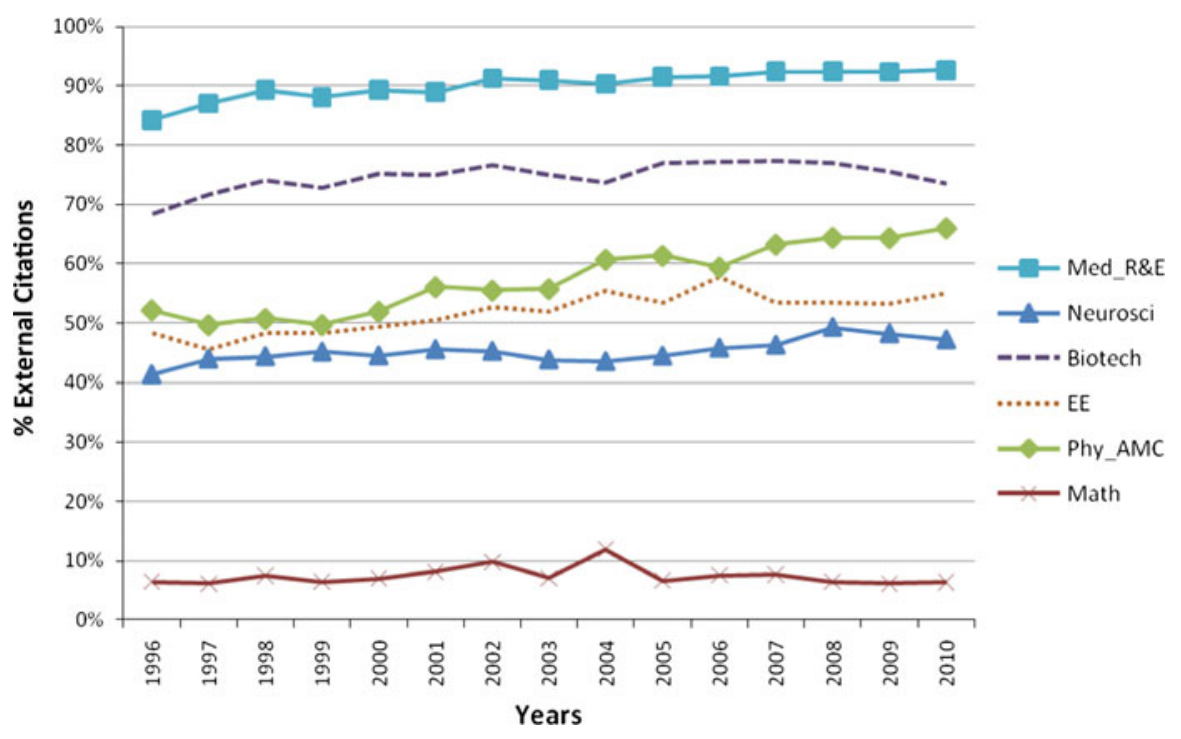

Fig. 3 Percentage external citations for 1995 benchmark sample articles over time

possibly yield insights into the basic mechanics of the diffusion process itself, is discussed below. Diffusion scores for most SCs seem to mildly increase over time since publication.

Before undertaking the effort required to calculate myriads of diffusion scores it might seem natural to wonder if a simpler approach would suffice. For example, might an analysis of external citations (i.e. citations from outside the field of analysis) measure a similar set of phenomena? Pursuant to this idea, Fig. 3 charts the percentage of external citations over time, for each of the 1995 benchmark SCs.

We note that each of the benchmarks exhibit a steep initial ascent over the first couple of years post-publication. We also see that the 1995 Math paper set has the lowest percentage of external citations (or highest percentage of internal cites). Also, the percentage of Math's external citations remains fairly constant over time. This finding is in keeping with trends in integration and diffusion scores for Mathematics. We notice a difference in the distance between the benchmarks for Figs. 2 and 3, as well as a greater degree of variation over time in the former figure, especially for the Math benchmark. Importantly, percentage of external citations does not inform us about the degree of variety, balance or diversity present in the citing article datasets (Stirling, 2007). Unique properties of the diffusion score are further elucidated below.

\section{Visualizing diffusion}

Subject overlay maps provide a complementary way to gain additional perspective on diffusion of research knowledge (Leydesdorff and Rafols 2009; Rafols et al. 2010; Rafols and Meyer 2010; Rafols and Leydesdorff 2009). These maps overlay SCs of articles citing our benchmark dataset on a base map of science. As was the case with the previous analyses, the cohorts in this section reflect citation data through the end of 2010. Lines connecting nodes reflect associations between SCs, with line thickness reflecting strength of association. Node sizes are proportional of the number of publications within a given 


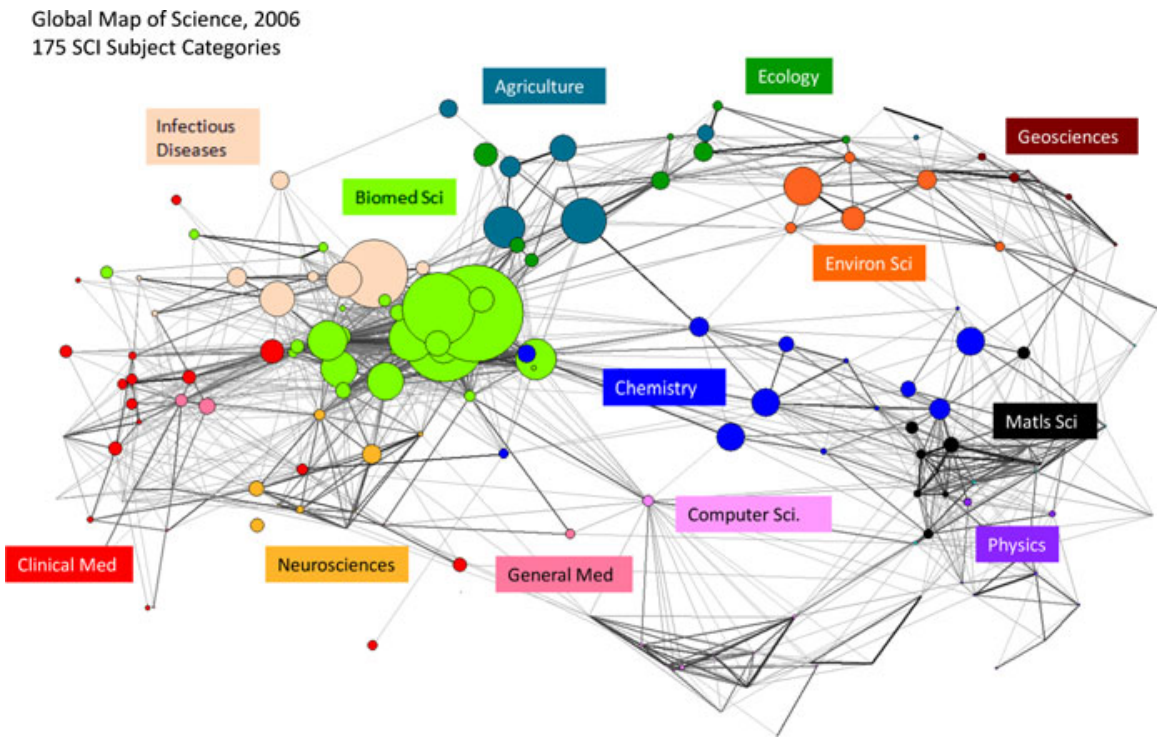

Fig. 4 Publications citing 1995 Biotechnology \& Applied Microbiology ("Biotech") benchmark sample articles

SC. This technique can be applied to visualize publication distribution for a given CSC cohort. The following analysis starts with a base map of SCs based on a year of crossjournal citation data. They build on the same year 2007 data covering the WOS SCI and SSCI used to generate the similarity matrix used in calculating the integration and diffusion scores. ${ }^{5}$ The labels in the maps are the Macro-disciplines from factor analyzing the SC-bySC cross-citations.

The science overlay maps locate research activity for particular SCs (i.e., based upon the journals in which papers appear). These maps offer a sense of the level of diffusion of a given benchmark (by the number of citing SCs on a given map), and they also impart a sense of which SCs cite a given benchmark with the greatest intensity (it is impossible to determine this from Figs. $2 \mathrm{a}$ or $2 \mathrm{~b}$ ). Figures 4, 5, 6, 7, 8 and 9 illustrate for our sample data. $^{6}$

From the preceding science overlay maps we observe that Fig. 6 (publications citing 1995 Math benchmark papers) is the most sparsely populated, and Fig. 7 (publications citing Med-R\&E benchmarks) is the busiest. This result is in keeping with Figs. 2a and $2 b$. We note also that Neuroscience seems to have the largest number of cites, shown by nodesize, from within its own field.

\footnotetext{
5 Loet Leydesdorff and Ismael Rafols have updated so that we now have a 2009 base map available at http://www.leydesdorff.net/overlaytoolkit. Ken Riopelle has generated a fine step-by-step process to produce one's own such maps (manual available at the previous website). We also offer papers on measuring and mapping interdisciplinarity, and an easy way to generate maps, at www.idr.gatech.edu/.

6 The macro-disciplines that appear in Figs. 4, 5, 6, 7, 8 and 9, which are mapped using a network visualization tool called Pajek, are color-coded and based on factor analysis. Vertices appear in Pajek's default size of 2.0 .
} 


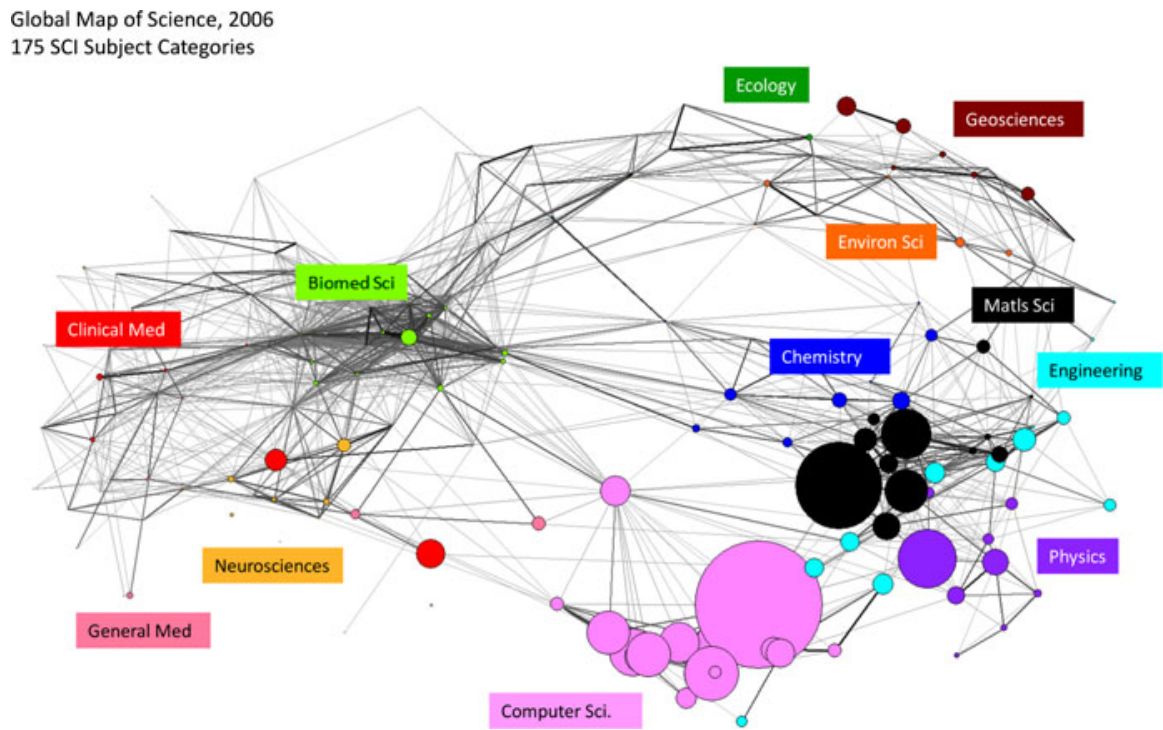

Fig. 5 Publications citing 1995 Engineering, Electrical \& Electronic("EE”) benchmark sample articles

Global Map of Science, 2006

$175 \mathrm{SCl}$ Subject Categories

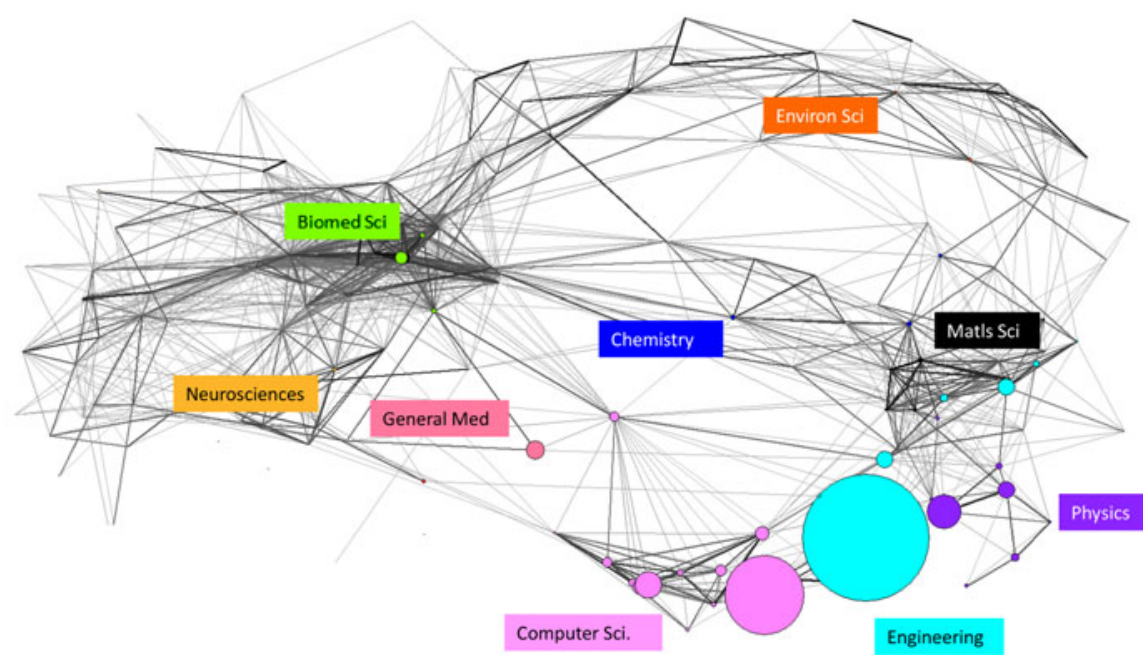

Fig. 6 Publications citing 1995 Mathematics benchmark sample articles

Mathematics: a subject category unto itself

From Fig. 2b we see that mean diffusion scores for medical R\&E, Neuroscience, Biotech, $\mathrm{EE}$, and Physics all generally reside within a range of 0.3 to 0.45 . Math, by contrast, has a mean diffusion score of 0.21 , and its mean annual diffusion scores seem to behave more 


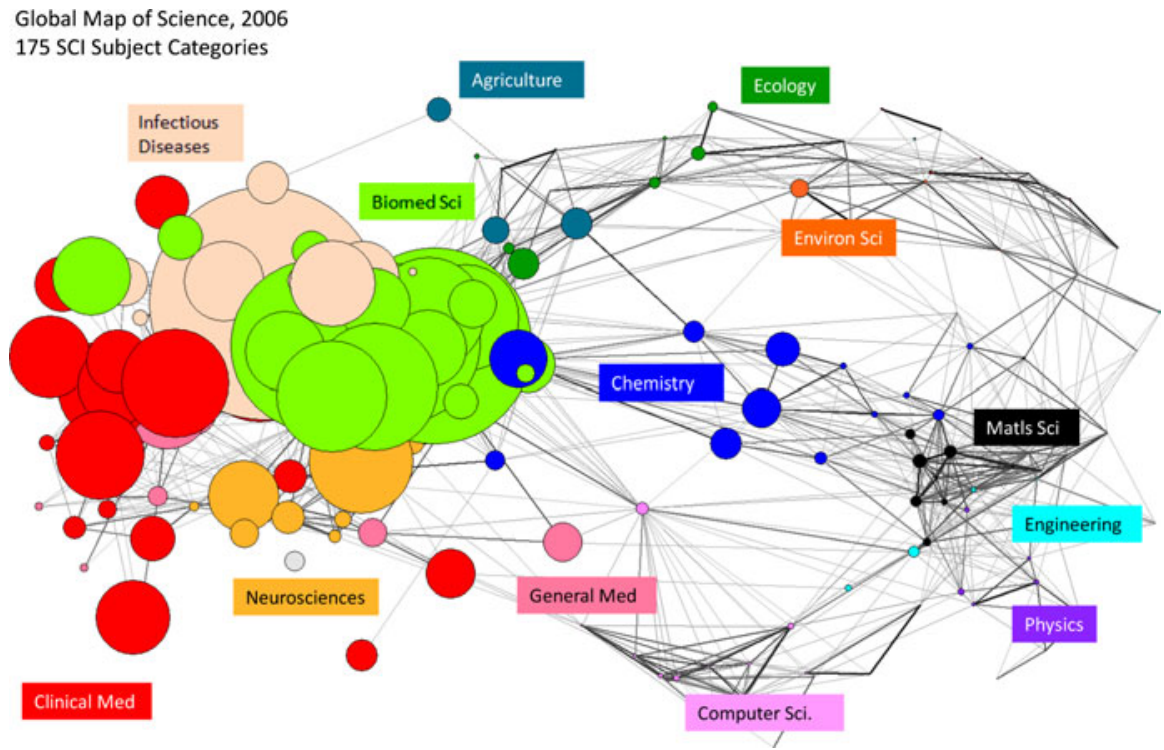

Fig. 7 Publications citing 1995 Medical Research \& Experimentation benchmark ("Med-R\&E") sample articles

Global Map of Science, 2006

$175 \mathrm{SCl}$ Subject Categories

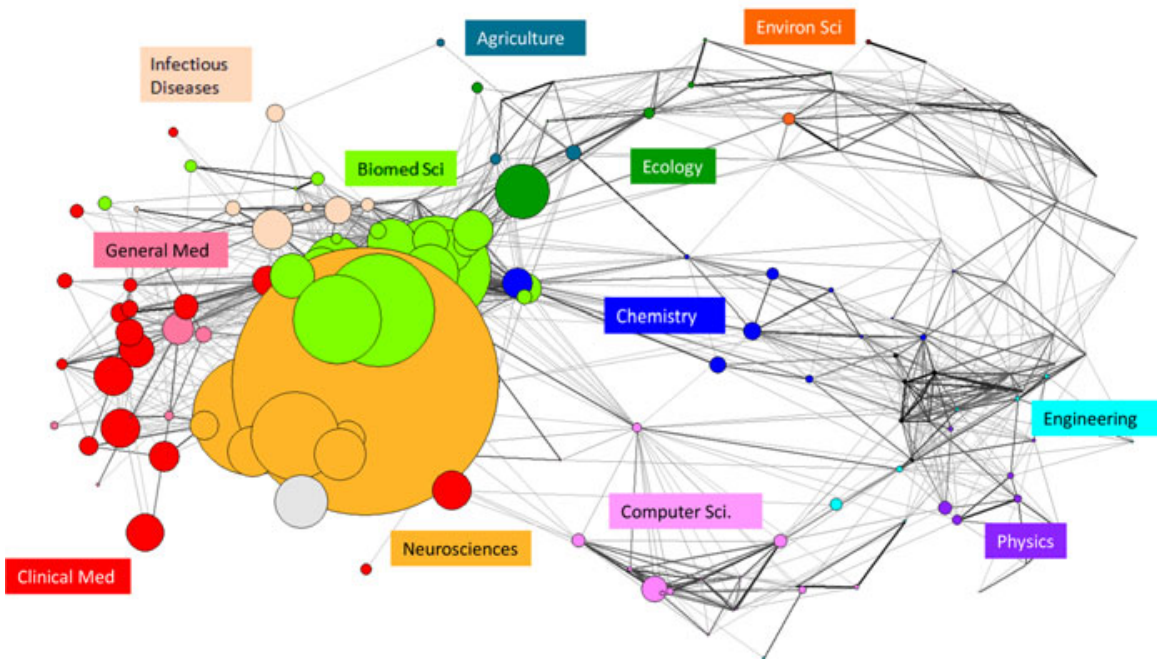

Fig. 8 Publications citing 1995 Neuroscience benchmark sample articles

erratically over time, especially in earlier years (i.e. 1996 to 2000). Math diffusion scores also appear to decline from 2005 onwards, whereas the other research areas edge slightly upward. We discuss possible interpretations for Math's unique behavior later

One might expect that if a given paper or discipline was cited more, that its Diffusion score would be higher, on average (i.e. aggregate trends for all benchmarks indicate that 
the more citations a given publication has, the more likely it is have diversity among its CSCs, on average - see Table 1). Using parallel logic, it might seem natural to equate higher cited reference counts with higher Integration scores. Exploring these hypotheses a bit further, Table 1 provides summary statistics for mean cited reference counts and times cited, and their correlation with integration and diffusion scores.

We observe from Table 1 that the correlation between diffusion and times cited is more consistent in terms of producing positive values than what we see between integration and cited references. However, the overall correlation coefficient for integration versus cited references (0.20) is larger than the same for diffusion versus times cited (0.13). The correlation coefficients (in the last two columns of Table 1) for individual benchmarks are not large (i.e. cited reference and times cited counts in the 1995 benchmark dataset do not wield a strong respective influence on integration and diffusion scores). The point is made that diffusion scores are calculated from a variety of inputs and cannot be estimated on the basis of citation counts. From Table 1 we see that citation rates among the benchmarks differ noticeably (i.e. the mean times cited is 59.72 for Med R\&E but 9.11 for Math). We also see that mean cited reference counts and times cited values are fairly similar for EE and Math. Hence, lower citation rates, in and of themselves, do not fully explain the differences in integration and diffusion scores between Math and the other SCs under consideration in this study.

To verify the normality of the above integration and diffusion scores we plotted their distribution for each benchmark SC dataset. Integration and diffusion score distributions for all of the benchmarks, except Math, assumed a bell shape. Most of the Math integration and diffusion score data-points, by contrast, concentrated at relatively low values. the sample distribution for Math was not normal. Because integration and diffusion scores for the sample of 658 Math papers, was not normally distributed, we conclude that the distribution of integration and diffusion scores for the larger population is not normally distributed either. Math is truly a unique field in terms of the distribution, size, and variation among its integration and diffusion scores.

To investigate factors underlying the behavior of Math diffusion scores, we queried several Mathematicians. One respondent, who requested anonymity, indicated that Math diffusion scores may be lower because higher-level research in Mathematics is particularly difficult for individuals without a strong quantitative background to understand. For this reason, Mathematicians are typically the only individuals who understand (and cite) one another. Scholars have noted, for example, that "Einstein's work did not receive immediate recognition due to its...Mathematical sophistication" (Small 2010). Moreover, the degree of precision required by Math also make this subject a difficult one for outsiders to understand and cite. Additionally, Math is an older discipline and its problems are very well-studied, making its higher-level research much more focused and/or refined. Surprising to us, the point was also made that Math papers are of longer than average length, ${ }^{7}$ making it again a more challenging discipline to approach from outside of its own boundaries. Finally, the case can be made that Math has more of a pure research focus, whereas the other SCs in this analysis are more applied in nature. These and similar factors may make Math citation patterns different than those of other disciplines.

\footnotetext{
7 The mean length for Math papers from our dataset was 19.43, which is significantly longer than Biotech (7.94), EE (8.15), Med-R\&E (8.11), Neuroscience (9.61) and Physics-AMC (9.42).
} 


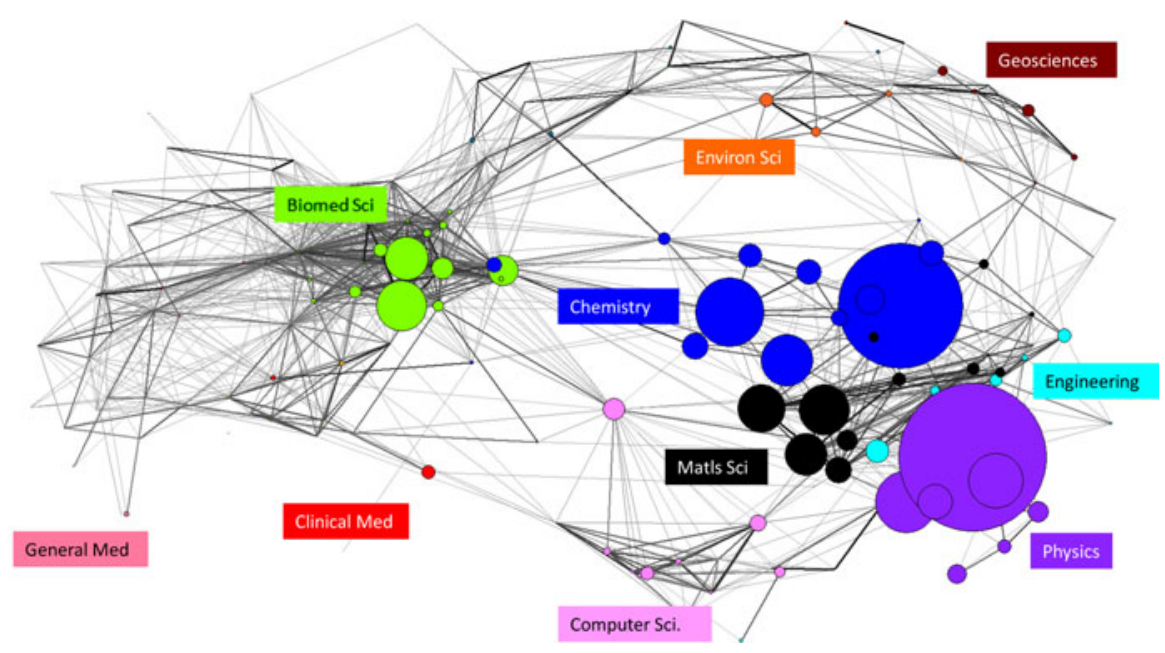

Fig. 9 Publications citing 1995 Physics, Atomic, Molecular \& Chemical ("Physics-AMC") benchmark sample articles

\section{Controlling for self-cites}

The preceding analyses calculated diffusion scores on the basis of all publications citing a given article. The question asked in this section, however, is: do self-citations impact diffusion scores? That is, how do diffusion scores change if we remove self-citations from total citations? To address this question we developed a macro that lists all authors for a given cited article, as well as all of the articles citing it. If any names appear in both lists, the citing article(s) affiliated with the common name is dropped from the dataset and a diffusion score is then calculated on the basis of the remaining articles.

Calculating diffusion scores without self-citations for the 1995 benchmark dataset produces interesting results. Before discussing these, however, we note that the difference in diffusion score between citing article datasets with and without self-cites is not expected to be overwhelming because (a) not all of the benchmark papers receive self-cites, and (b) among those that do, self-cites usually do not constitute a large percentage of total cites. Because of its unique diffusion properties, we discuss the influence of self-cites on the Math 1995 dataset first.

Figure 10 plots average annual diffusion scores, with and without self-cites, for the Math 1995 benchmark dataset. We see that when self-cites are removed, mean annual diffusion scores increase, on average. More specifically: mean annual diffusion scores increased for 12 of the 15 years plotted, held steady for one year, and decreased (by 0.01 ) for two of the years shown in Fig. 10. We note that the preponderance of the impact of selfcitations for the Math 1995 dataset was greatest in the first four years and began to taper off after that. Mean diffusion scores increase, on average, when self-cites are removed because Math articles tend to get cited primarily by other Math articles (see Fig. 3), and if a given Math publication is already being cited by other Math publications, removing a self-cite will not meaningfully influence SC diversity among the publication's citing article dataset 


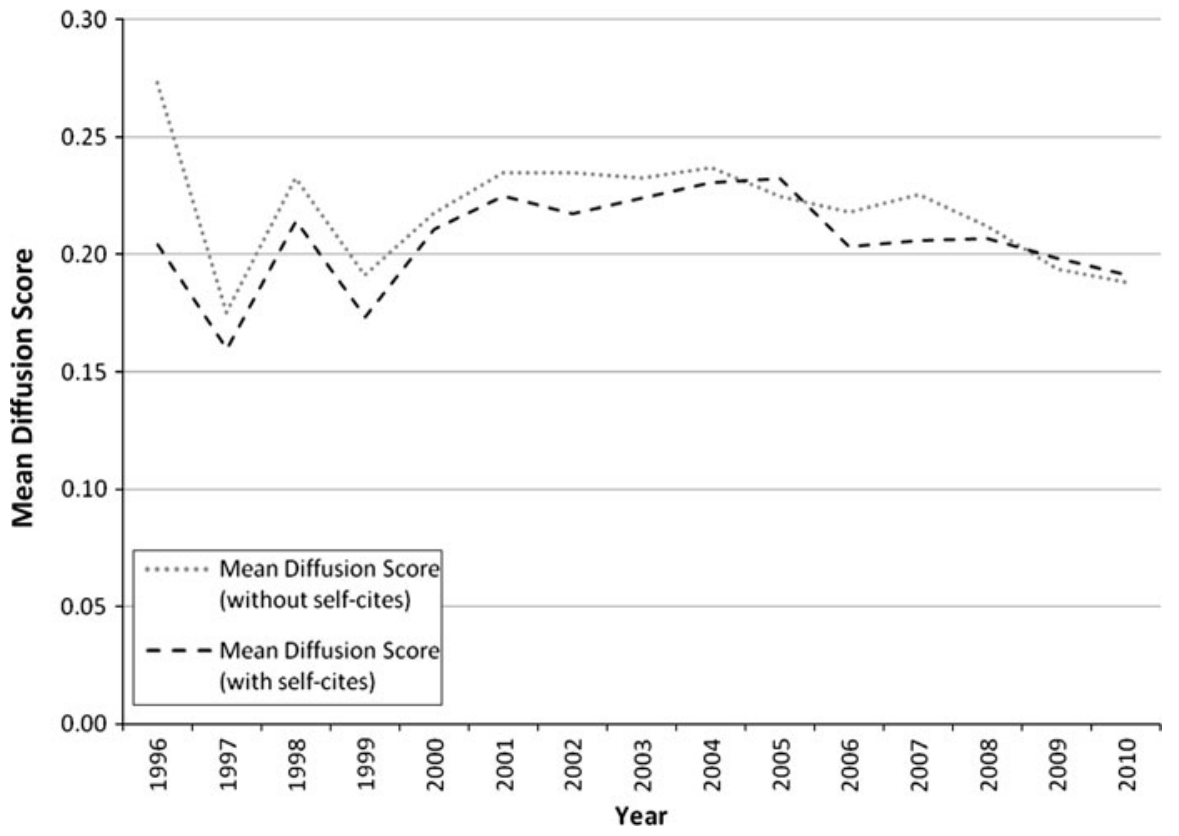

Fig. 10 Mean annual 1995 Math diffusion scores

(e.g. if a Math publication was cited by four Math publications, including itself, and one Physics publication, dropping self-cites would not radically alter diversity among CSCs). The removal of self-cites will, however, decrease the total number of citing article instances. Hence, when self-cites for Math are dropped, the ratio of SC diversity to total cites (i.e. SC diversity per article for the citing article dataset) will increase, on average, putting upward pressure on mean diffusion scores. We note, however, that the mean Diffusion score for all years plotted in Fig. 10 is 0.21 with self-cites and 0.22 without-a modest overall increase.

As can be seen in Figs. 2 and 3, Med R\&E and Math 1995 benchmarks fall at the opposite end of citation and diffusion spectrums. We therefore next calculate mean annual diffusion scores, with and without self-citations, for 1995 Med R\&E benchmarks. These appear in the following figure.

We see that the two lines in Fig. 11 almost perfectly overlap, indicating that the influence of self-cites on mean diffusion scores for this dataset is miniscule. We note that self-citations form a small fraction of total citations here, and that after self-cites are removed the SC diversity among the citing article dataset is relatively unaffected. Because this outcome is typical of the remaining benchmarks, we do not present similar figures for them here.

\section{Discussion}

In this study, we have introduced a new metric for measuring the propagation of knowledge. It incorporates Stirling's (2007) notions of variety, balance and disparity among 


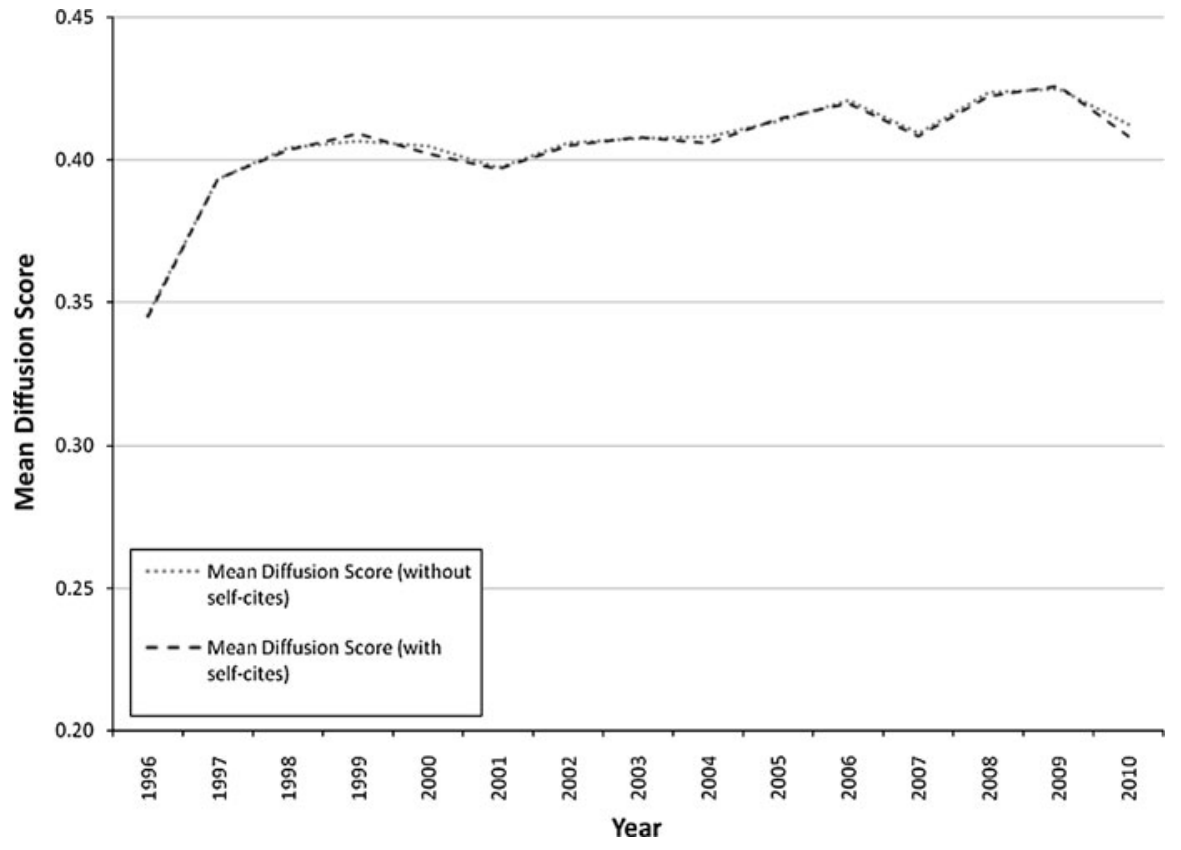

Fig. 11 Mean annual 1995 Med R\&E diffusion scores

citing article cohorts. This provides a richer depiction of dispersion than do simpler measures, such as percentage of citations from outside the field of the journal in which an article is published.

This diffusion score is analogous to Integration score in its formulation. Integration score has proven to be a useful metric in evaluation of National Academy Keck Futures Initiative activities. It has also been helpful in gauging the research outputs of funding support that purports to be interdisciplinary:

- National Science Foundation (NSF) Human and Social Dynamics Priority Area (Garner and Porter, to appear)

- NSF Research on Learning and Education Program (Porter et al. 2010)

- NSF Research Cooperation Networks (Garner et al., under revision)

- National Institutes of Health Transdisciplinary Tobacco Use Research Centers (TTURC) Program (internal report)

Yegros-Yegros et al. (2010) used Integration scoring in studying whether such papers have higher impact (we would now suggest calculating the Diffusion score as part of such investigation). We offer this relatively simple metric as a new research assessment aid.

In this paper we report on the formulation of diffusion score and on initial applications. We focus on "benchmark" sample article sets to help establish the behavior of this measure. In the process, we find quite interesting results in their own right. Diffusion score differences among the six SC sample sets studied manifest not only in absolute terms, but also in terms of rates of change, variance and movements with interrelated benchmark variables. 
For most SCs, diffusion scores increase with the passage of time (at least for our 1995 benchmark dataset), Math behaving differently. Diffusion results for Math challenge the image of it as a "foundational research area." That image would have others citing Math relatively more than Math cites other fields. Here we observe Math tending to both mainly cite research in Math or closely associated fields, and to be cited mainly by papers in Math or closely associated fields. Other fields do not draw very heavily on Math research, at least directly. It could be interesting to pursue further analyses to see if one can track significant "second generation" citing (e.g., fields like statistics or operations research heavily cite Math, and then other fields heavily cite them).

We hope that other researchers will explore the behavior and merits of the diffusion score. We recognize that issues abound. Table 1 calls attention to differences in citation intensity among the six SCs studied. Research has shown that citation motivation and practices are highly field dependent (Li and Meng 2010), making field normalization important for fair comparison of results in bibliometric studies (Zitt et al. 2005). Research has also shown that different journals have different average citation counts per paper, pointing to the need for the normalization of journal citation impact measures (Moed 2010). Study of the behavior of Integration and diffusion scores for a wider set of research fields, possibly enriched by visualizations such as science overlay maps, would be valuable. In-depth analyses of selected papers, possibly enriched by discussion with the authors, could illuminate the bases of integration and diffusion scores.

\section{Appendix A: WOS subject category descriptions ${ }^{8}$}

Biotechnology and applied microbiology

Description Biotechnology and applied microbiology includes resources that cover a broad range of topics on the manipulation of living organisms to make products or solve problems to meet human needs. Topics include genetic engineering; molecular diagnostic and therapeutic techniques; genome data mining; bioprocessing of food and drugs; biological control of pests; environmental bioremediation; and bio-energy production. This category also covers resources that deal with the related social, business, and regulatory issues.

Engineering, electrical and electronic

Description Engineering, electrical and electronic covers resources that deal with the applications of electricity, generally those involving current flows through conductors, as in motors and generators. This category also includes resources that cover the conduction of electricity through gases or a vacuum as well as through semiconducting and superconducting materials. Other relevant topics in this category include image and signal processing, electromagnetics, electronic components and materials, microwave technology, and microelectronics.

\footnotetext{
${ }^{8}$ As they appear in: http://science.thomsonreuters.com/mjl/scope/scope_scie/
} 


\section{Mathematics}

Description Mathematics covers resources having a broad, general approach to the field. The category also includes resources focusing on specific fields of basic research in Mathematics such as topology, algebra, functional analysis, combinatorial theory, differential geometry and number theory.

Medicine, research and experimental

Description Medicine, research and experimental includes resources describing general medical research with a particular emphasis on extremely novel techniques and clinical interventions in a broad range of medical specializations and applications, including vaccine development, tissue replacement, immunotherapies, and other experimental therapeutic strategies. Resources in this category reflect clinical interventions that are in early stages of development, using in vitro or animal models, and small-scale clinical trials.

\section{Neurosciences}

Description Neurosciences covers resources on all areas of basic research on the brain, neural physiology, and function in health and disease. The areas of focus include neurotransmitters, neuropeptides, neurochemistry, neural development, and neural behavior. Coverage also includes resources in neuro-endocrine and neuro-immune systems, somatosensory system, motor system and sensory motor integration, autonomic system as well as diseases of the nervous system.

Physics, atomic, molecular and chemical

Description Physics, atomic, molecular and chemical includes resources concerned with the Physics of atoms and molecules. Topics covered in this category include the structure of atoms and molecules, atomic and molecular interactions with radiation, magnetic resonances and relaxation, Mossbauer effect, and atomic and molecular collision processes and interactions.

\section{Appendix B: Cited papers within each benchmark that have at least 3 CSCs}

We note from this figure that Math, once again, is the outlier. The remaining five benchmarks display a similar pattern: they increase modestly from for the first citing year or two, and then steadily decrease from that point onward (i.e. these benchmarks tend to get cited less over time). Figure 12a plots data for individual citing years. If we include all citing years for each benchmark, however, a different picture emerges (see Fig. 12b).

We note from this figure that the percentage of cited papers with at least 3 CSCs is noticeably higher than what is seen in Fig. 12a. This can be attributed to the fact that, when all citing years are included, cited papers, on average, have more papers (and hence more CSC instances) citing them. 

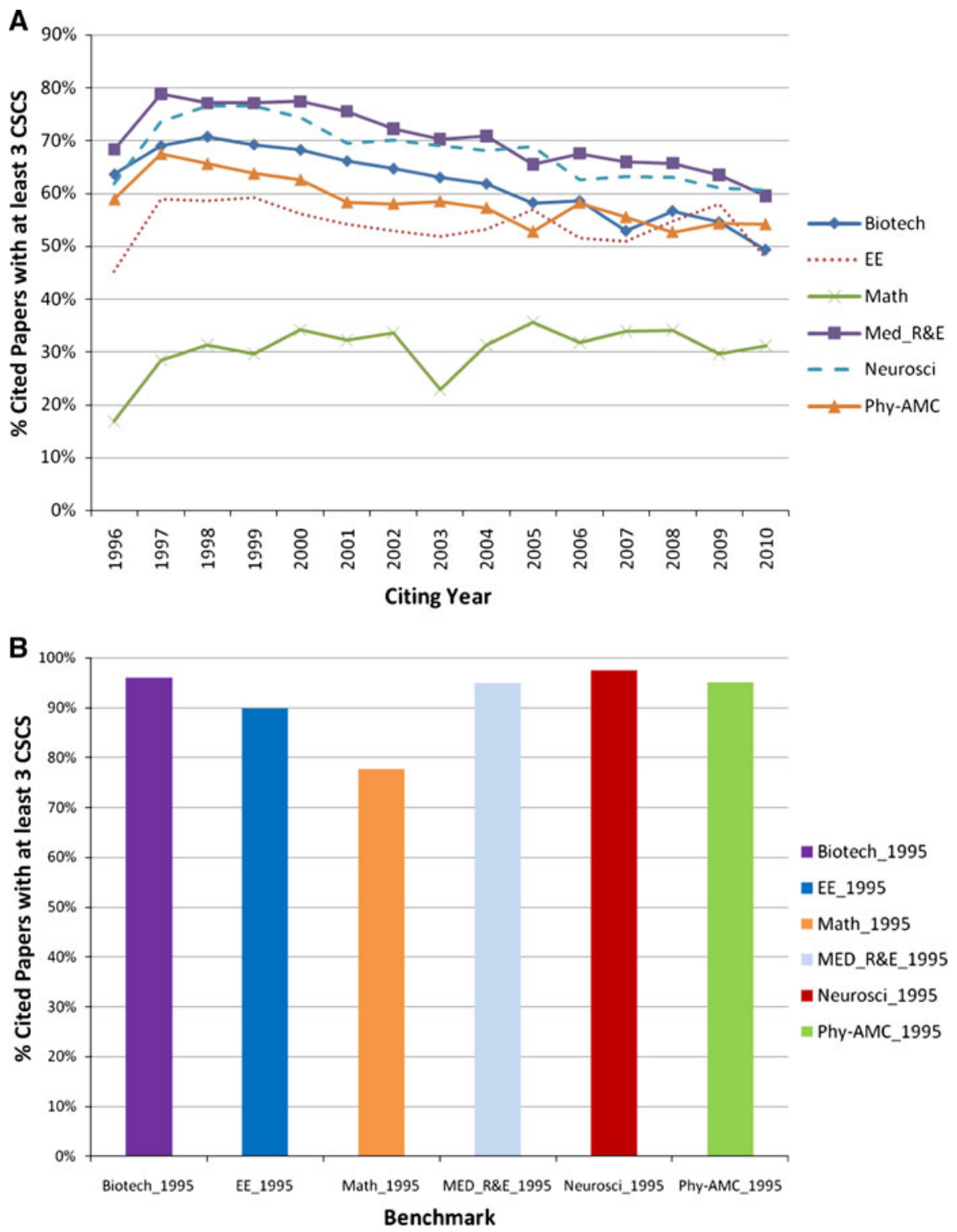

Fig. 12 a Percentage of cited papers with at least 3 CSC instances, by benchmark, by citing year. b Percentage of cited papers with at least 3 CSC instances, by benchmark, for all citing years

\section{References}

Autant-Bernard, C., Mairesse, J., \& Massard, N. (2007). Spatial knowledge diffusion through collaborative networks. Papers in Regional Science, 86, 341-350.

Bacchiocchi, E., \& Montobbio, F. (2009). Knowledge diffusion from university and public research. A comparison between US, Japan and Europe using patent citations. Journal of Technology Transfer, 34(2), 169-181. 
Birnbaum-More, P. H., Rossini, F. A., \& Baldwin, D. R. (Eds.). (1990). International research management. New York: Oxford University Press.

Chen, C., Chen, Y., Horowitz, M., Hou, H., Liu, Z., \& Pellegrino, D. (2009). Towards an explanatory and computational theory of scientific discovery. Journal of Informetrics, 3(3), 191-209.

Chen, C., \& Hicks, D. (2004). Tracing knowledge diffusion. Scientometrics, 59(2), 199-211.

Chubin, D. E., \& Connolly, T. (1982). Research trails and science policies: Local and extra-local negotiations of scientific work. In N. Elias, H. Martins, \& R. Whitley (Eds.), Scientific establishments and hierarchies. Sociology of the sciences, yearbook (Vol. 6, pp. 293-311). Dordrecht: D. Reidel.

Chubin, D. E., Rossini, F. A., Porter, A. L., \& Connolly, T. (Eds.). (1986). Interdisciplinary analysis and research. Mt. Airy, MD: Lomond.

De Bellis, N. (2009). Bibliometrics and citation analysis: From the science citation index to cybermetrics. Lanham, MD: Scarecrow Press.

Drexler, K.E. (1986). Engines of creation: The coming era of nanotechnology, New York: Anchor Books; available free at http://e-drexler.com/p/06/00/EOC_Cover.html.

Garner, J., Porter, A.L. (to appear). Assessing the human and social dynamics program-exceptional crossdisciplinarity. In Proceedings of Atlanta conference on science and innovation policy 2011 (September 14-17). Atlanta, USA.

Garner, J., Porter, A.L., Newman, N.C., Crowl, T. (under revision), Assessing Research Network and Disciplinary Engagement Changes Induced by an NSF Program, Research Evaluation.

Jurkovick, R., \& Paelincer, J. H. P. (Eds.). (1984). Problems in interdisciplinary studies. Aldershot, England: Gow Publishing.

Katz, J. S., Hicks, D. M. (1995). The classification of interdisciplinary journals: A new approach. In Proceedings of the fifth biennial conference of the international society for scientometrics and infometrics, Rosary College, ILL (pp. 245-255). Medford, NJ: Learned Information, Inc.

Klavans, R., \& Boyack, K. W. (2009). Toward a consensus map of science. Journal of the American Society for Information Science and Technology, 60(3), 455-476.

Klein, J. T. (1996). Crossing boundaries: Knowledge, disciplinarities, and interdisciplinarities. Charlottesville, VA: University Press of Virginia.

Leydesdorff, L., \& Rafols, I. (2009). A global map of science based on the ISI subject categories. Journal of the American Society for Information Science and Technology, 60(2), 348-362.

Li, R., \& Meng, L. (2010). On the framing of patent citations and academic paper citations in reflecting knowledge linkage: A discussion of the discrepancy of their divergent value-orientations. Chinese Journal of Library and Information Science, 3(3), 37-45.

Liu, Y. X., \& Rousseau, R. (2010). Knowledge diffusion through publications and citations: A case study using ESI-fields as unit of diffusion. Journal of the American Society for Information Science and Technology, 61(2), 340-351.

MacGarvie, M. (2005). The determinants of international knowledge diffusion as measured by patent citations. Economics Letters, 87, 121-126.

Masse, L.C., Moser, R.P., Stokols, D., Taylor, B.K., Marcus, S.E., Morgan, G.D., Hall, K.L., Croyle, R.T., Trochim, W.M. (2008). Measuring collaboration and transdisciplinary integration in team science. American Journal of Preventive Medicine, 35 (2): S151-S160.

Moed, H. (2010). Measuring contextual citation impact of scientific journals. Journal of Informetrics, 4(3), $265-277$.

National Academies - Committee on Science, Engineering, and Public Policy (COSEPUP) Committee on Facilitating Interdisciplinary Research (2005). Facilitating interdisciplinary research. Washington, DC: National Academies Press.

Porter, A.L., Rafols, I (2009b). Measuring and mapping interdisciplinarity in six research fields over time (1975-2005). In ISSI Conference, Rio de Janeiro, Brazil.

Porter, A. L., Cohen, A. S., Roessner, J. D., \& Perreault, M. (2007). Measuring researcher interdisciplinarity. Scientometrics, 72(1), 117-147.

Porter, A. L., \& Cunningham, S. (1995). Whither nanotechnology? A bibliometric study. Foresight Update, $21,12-15$.

Porter, A. L., \& Rafols, I. (2009a). Is science becoming more interdisciplinary? Measuring and mapping six research fields over time. Scientometrics, 81(3), 719-745.

Porter, A. L., Roessner, J. D., Cohen, A. S., \& Perreault, M. (2006). Interdisciplinary research-meaning, metrics and nurture. Research Evaluation, 15(3), 187-195.

Porter, A. L., Roessner, J. D., \& Heberger, A. E. (2008). How interdisciplinary is a given body of research? Research Evaluation, 17(4), 273-282.

Porter, A. L., Schoeneck, D. J., Roessner, D., \& Garner, J. (2010). Practical research proposal and publication profiling. Research Evaluation, 19(1), 29-44. 
Rafols, I., \& Leydesdorff, L. (2009). Content-based and algorithmic classifications of journals: Perspectives on the dynamics of scientific communication and indexer effects. Journal of the American Society for Information Science and Technology, 60(9), 1823-1835.

Rafols, I., \& Meyer, M. (2010). Diversity and network coherence as indicators of interdisciplinarity: case studies in bionanoscience. Scientometrics, 82(2), 263-287.

Rafols, I., Porter, A. L., \& Leydesdorff, L. (2010). Science overlay maps: A new tool for research policy and library management. Journal of the American Society for Information Science \& Technology, 61(9), 1871-1887.

Singh, J. (2005). Collaborative networks as determinants of knowledge diffusion patterns. Management Science, 51, 756-770.

Small, H. (2010). Referencing through history: How the analysis of landmark scholarly texts can inform citation theory. Research Evaluation, 19(3), 185-193.

Stirling, A. (2007). A general framework for analysing diversity in science, technology and society. Journal of the Royal Society Interface, 4(15), 707-719.

Stokols, D., Fuqua, J., Gress, J., Harvey, R., Phillips, K., Baezconde-Garbanati, L., et al. (2003). Evaluating transdisciplinary science. Nicotine and Tobacco Research, 5(Suppl. 1), S21-S39.

Stolpe, M. (2002). Determinants of knowledge diffusion as evidenced in patent data: the case of liquid crystal display technology. Research Policy, 31(7), 1181-1198.

Wagner, C. S., Roessner, J. D., Bobb, K., Klein, J. T., Boyack, K. W., Keyton, J., et al. (2011). Approaches to understanding and measuring interdisciplinary scientific research (IDR): A review of the literature. Journal of Informetrics, 5(1), 14-26.

Yegros-Yegros, A., Amat, C., D;Este, P., Porter, A.L., Rafols, I. (2010, September). Does interdisciplinary research lead to higher impact? STI Conference (Science \& Technology Indicators), Leiden.

Yu, G., Wang, M. Y., \& Yu, D. R. (2010). Characterizing knowledge diffusion of nanoscience \& nanotechnology by citation analysis. Scientometrics, 84(1), 81-97.

Zitt, M., Ramanana-Rahary, S., \& Bassecoulard, E. (2005). Relativity of citation performance and excellence measures: From cross-field to cross-scale effects of field-normalisation. Scientometrics, 63(2), 373-401. 